\title{
MintHint: Automated Synthesis of Repair Hints
}

\author{
Shalini Kaleeswaran Varun Tulsian Aditya Kanade \\ Indian Institute of Science \\ \{shalinik,varuntulsian,kanade\}@csa.iisc.ernet.in
}

\author{
Alessandro Orso \\ Georgia Institute of Technology \\ orso@cc.gatech.edu
}

\begin{abstract}
Being able to automatically repair programs is at the same time a very compelling vision and an extremely challenging task. In this paper, we present MintHint, a novel technique for program repair that is a departure from most of today's approaches. Instead of trying to fully automate program repair, which is often an unachievable goal, MintHint performs statistical correlation analysis to identify expressions that are likely to occur in the repaired code and generates, using pattern-matching based synthesis, repair hints from these expressions. Intuitively, these hints suggest how to rectify a faulty statement and help developers find a complete, actual repair. MintHint can address a variety of common faults, including incorrect, spurious, and even missing expressions.

We also present an empirical evaluation of MintHint that consists of two main parts. The first part is a user study that shows that, when debugging, developers' productivity can improve manyfold with the use of repair hints-compared to having only traditional fault localization information. The second part consists of applying MintHint to several faults of a widely used Unix utility program to further assess the effectiveness of the approach. Our results show that MintHint performs well even in situations, seen frequently in practice, where (1) the repair space searched does not contain the exact repair, and (2) the operational specification obtained from the test cases for repair is incomplete or even imprecise-which can be challenging for approaches aiming at fully automated repair.
\end{abstract}

\section{Introduction}

Debugging is an expensive activity that can be responsible for a significant part of the cost of software maintenance [39]. It is therefore not surprising that researchers and practitioners alike have invested a great deal of effort in developing techniques that can improve the efficiency and effectiveness of debugging (e.g., [3] 6 , 7 . 11, 15, 25, 29, 31, 32, 34, 35, 42 I). In particular, in recent years, there has been a growing interest in automated program repair techniques (e.g., [6, 11, 29, 31, 32, 35]). Although these techniques have been shown to be effective, they suffer from one or more of the following limitations. First, some techniques rely on the existence of a specification for the program being debugged, which is rarely the case in practice. Second, techniques that do not rely on specifications tend to "overfit" the repair to the set of existing test cases, which is likely to affect the general validity of the repair. Third, because they are looking for a complete repair, most existing techniques must perform a search over a repair space that is large enough to include the (unknown) repair. For non-trivial repairs, this can make the technique either ineffective (if the bound on the repair space used by the tool is too small) or too expensive to be used in practice (if the bound used/required is too large).

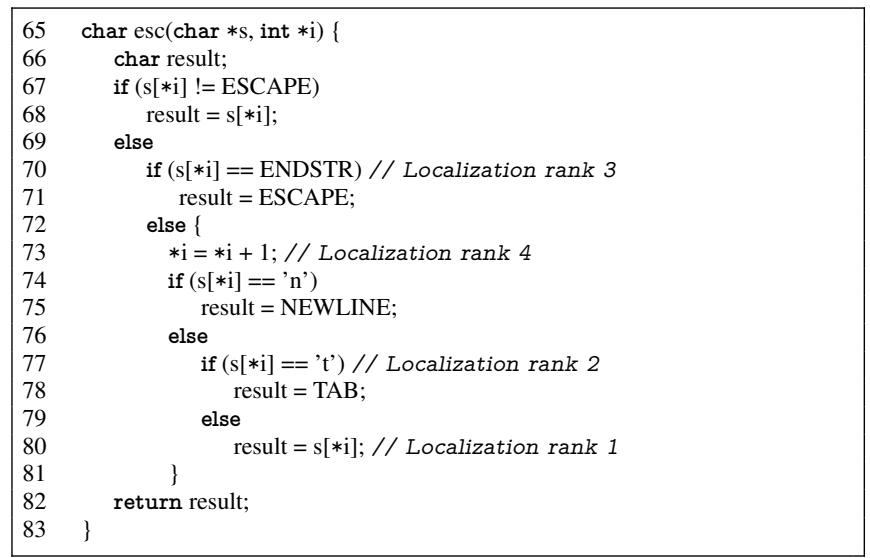

Figure 1: Function esc from program replace (version 23), which contains a faulty statement at line 70 . The ones shown are the line numbers of function esc in the actual code.

To address these limitations of existing techniques, in this paper we propose MintHint, a novel, semi-automated approach to program repair. MintHint is a departure from most of today's program repair techniques as it does not try to find a complete repair, which we have observed to be an unachievable goal in many, if not most, cases due to technical and practical reasons. Instead, MintHint aims to generate repair hints that suggest how to rectify a faulty statement and help developers find a complete, actual repair.

As an example, consider function esc from a faulty version of program replace [19], shown in Figure 1] Function esc takes as input a string and an index into the string and checks whether the character at the index is a special character (such as a newline or a tab). If so, it returns a program-specific constant that represents the special character. The fault is at line 70 , where the branch predicate should be $s\left[{ }^{*}+1\right]==$ ENDSTR, but an incorrect array index, ${ }^{*} \mathrm{i}$, is used instead. Given this faulty program and a set of test cases for the program that trigger the fault (i.e., at least one test in the set fails due to this fault), MintHint would produce the following hint:

$$
\text { Replace } s\left[{ }^{*} i\right]==\text { ENDSTR by } s\left[{ }^{*} i+1\right]==\text { ENDSTR }
$$

Developers would use this hint as guidance while modifying the original code to arrive at a repair. In this specific example, developers would simply make the suggested change and obtain the repaired version. In the more general case, as we will show in Section 4 hints are not necessarily complete repairs, but rather suggestions on how to generate such repairs.

In the common case in which more than one statement is suspected to be faulty, MintHint would algorithmically generate repair hints for all of them. It would then rank the generated hints to help developers prioritize their efforts. 
To generate hints, MintHint operates in four steps. The first step identifies potentially faulty statements by leveraging an existing fault localization technique that requires only a test suite (e.g., [1, 20, 25]). The subsequent steps are performed for each of the identified statements. The second step derives a state transformer, that is, a function that (1) is defined for all program states that reach the faulty statement in the given test suite and (2) produces the right output state for each of them. This step leverages dynamic symbolic execution techniques (e.g., [4]). The third step explores a repair space and tries to identify and rank, through a statistical correlation analysis [8, 27], expressions in the space that are likely to occur in the repaired statement, using the state transformer derived in the second step. (To the best of our knowledge, this paper is the first one to apply this form of statistical reasoning to programs.) Finally, the fourth step of the approach synthesizes repair hints by pattern matching [41] the expressions computed in the previous step with those in the faulty statement. Together these steps result in a sophisticated technique that suitably combines symbolic, statistical, and syntactic reasoning to help in program repair.

MintHint synthesizes five types of hints that suggest (1) insertion, (2) replacement, (3) removal, or (4) retention of expressions, and (5) combinations of these. As shown in Table 1 these hints are applicable to many types of common faults, ranging from incorrect or spurious expressions to missing expressions, to combinations of these. Moreover, MintHint can handle faults in a variety of program constructs, such as assignments, conditionals, switch statements, loop headers, return statements, and statements with ternary expressions. The main restriction, for the technique presented in this paper, is that the fault has to involve a single statement and, in the case of an assignment, it must be on the right-hand side (RHS) expression. However, we believe that MintHint can be extended to address other situations, e.g., where the left-hand side variable in an assignment is faulty or the fault spans multiple statements.

MintHint overcomes the main limitations of existing techniques that we listed earlier in the following ways. First, it does not rely on a formal specification; it instead derives an operational specification (i.e., a state transformer) from the test cases available. Second, approaches that aim at deriving complete repair, typically use equality with the state transformer (or an analogous entity) as a criterion for selecting a candidate repair (e.g., [29 31]). The statistical correlation used in MintHint is a more relaxed and robust notion than equality and can thus be more effective in identifying which expressions are likely to be part of the repaired code; this allows MintHint to generate more general repairs and to be effective in the presence of incomplete or even imperfect (i.e., noisy) data. Third, since MintHint looks for building blocks of repair (rather than the complete repair itself) and then combines them algorithmically to generate compound hints, it can generate useful actionable hints even when exploring an incomplete repair space.

To evaluate MintHint, we developed a prototype tool that implements our approach for C programs and performed a user study using programs from the Siemens benchmark [19]. Specifically, the study consisted of two phases: control and experimental. In both phases, a user was provided a single repair task along with fault localization information and a test suite. In the experimental phase, in addition, repair hints generated by MintHint were supplied. The tasks given to a user in the two phases were independent. In all 10 users were involved in the study. Without repair hints, only 6 of them completed their task within $2 \mathrm{~h}$. With repair hints, all users could complete their task within the same time limit. For example, the fault described in Figure 1 was part of the user study but without hints the user could not repair it within $2 \mathrm{~h}$, whereas with hints another user repaired it easily. Moreover, for the tasks completed

\begin{tabular}{|l|l|}
\hline Nature of hint & Targeted fault \\
\hline Insert & Missing expressions \\
\hline Replace & Incorrect operator, constant, variable, etc. \\
\hline Remove & Spurious expressions \\
\hline Retain & Eliminating false positives \\
\hline Compound & One or more occurrences of above \\
\hline
\end{tabular}

Table 1: Nature of hints and targeted faults.

in both the phases, in the experimental phase, with hints, they were completed over 5 times faster.

In addition to the user study, we also performed a case study using a commonly used Unix utility, namely, the stream editor sed. We considered 6 faulty versions of sed. On one of them symbolic execution timed out and hence it was not subjected to hint generation. On 3 of the remaining 5 faulty versions, MintHint provided hints that immediately lead to repair. In one additional case, the hints when applied to the program, resulted in a partial repair in which all passing tests continued to pass but several failing tests started passing. Further, MintHint was able to synthesize useful hints even in the many cases across the user study and the case study in which (1) the repair space considered did not contain the repaired version of the faulty expression, or (2) the state transformer contained imperfect data.

In summary, the main contributions of this paper are:

- The definition of a novel technique for program repair, called MintHint, that combines symbolic, statistical, and syntactic reasoning and overcomes some of the main limitations of existing techniques by focusing on generating repair hints, rather than complete repairs.

- An implementation of MintHint that can perform automated synthesis of repair hints for $\mathrm{C}$ programs.

- A user study that evaluates the effect of repair hints on developers' productivity. This is one of the few user studies performed in the area of program repair and debugging in general.

- A further evaluation of MintHint's effectiveness on a case study involving several faulty versions of a Unix utility.

\section{Overview}

Figure 2 provides a high-level view of MintHint. As the figure shows, MintHint takes as input a faulty program and a test suitewhere at least one test case triggers the fault in the program, and thus fails - and produces a list of repair hints in four steps. We now describe these four steps using the example we discussed in the Introduction, shown in Figure 1 .

Step 1: Fault localization. This is a preliminary step, whose goal is to provide the hint generation algorithm with a list of possibly faulty statements to be repaired. To compute this list, MintHint can leverage any existing fault localization approach. In our current implementation, we use the Ochiai approach as implemented in the Zoltar tool [20], which uses the test suite and performs spectrabased fault localization. For our example, the localization tool outputs a ranked list of suspicious statements: $80,77,70,73,502$, and so on. In its subsequent steps, MintHint runs the hint generation algorithm on each statement in the fault localization list independently, up to a given threshold. In the following discussion, we use line 70 to illustrate the remaining steps of MintHint.

Step 2: Derivation of state transformers. The next step towards repair is to infer the specification of what would be the correct statement at line 70. To do so, in the absence of an actual specification, MintHint uses the test suite provided for the program to infer an operational specification for the statement in the 


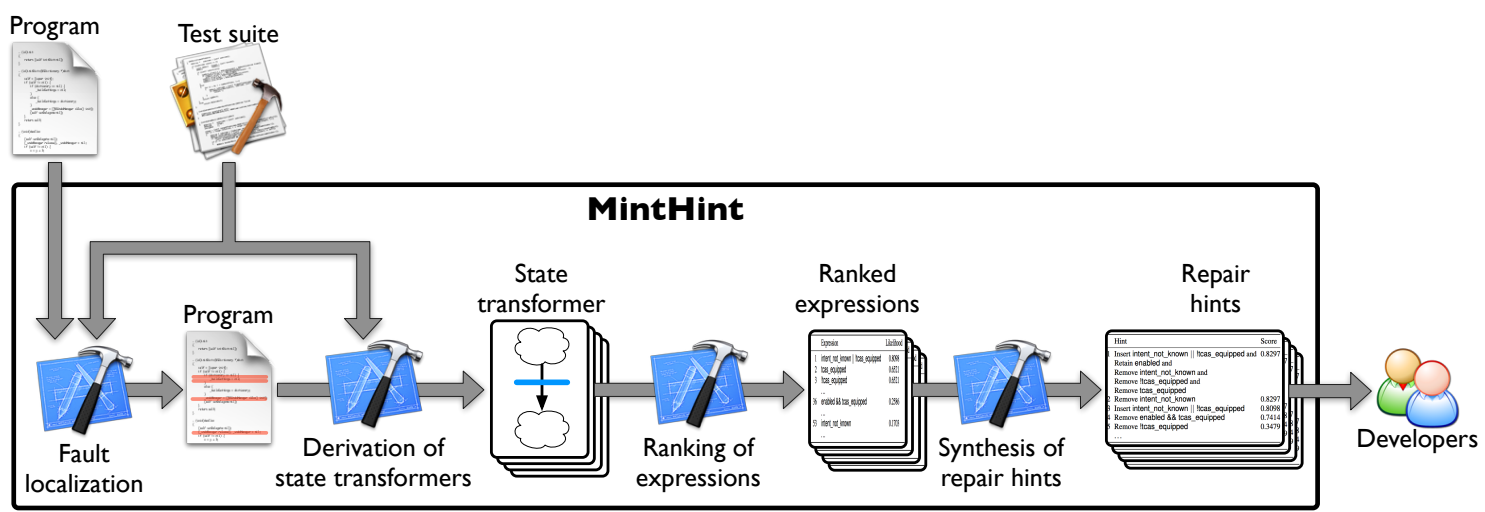

Figure 2: High-level view of the MintHint approach.

\begin{tabular}{|c|c|c|c|c|c|}
\hline \multicolumn{2}{|c|}{ Input state } & \multirow{2}{*}{$\frac{\text { Output state }}{\text { branch }_{0}}$} & \multicolumn{3}{|c|}{ Values of expressions over input state } \\
\hline $\mathrm{s}$ & ${ }^{*} \mathrm{i}$ & & $\begin{array}{l}\mathrm{s}\left[{ }^{*} i\right]== \\
\mathrm{s}\left[{ }^{*} \mathrm{i}+1\right]\end{array}$ & $\begin{array}{c}\mathrm{s}\left[{ }^{*} \mathrm{i}\right]== \\
\text { ENDSTR }\end{array}$ & $\begin{array}{l}\mathrm{s}\left[{ }^{*} \mathrm{i}+1\right]== \\
\text { ENDSTR }\end{array}$ \\
\hline "@\%\&a" & 0 & false & false & false & false \\
\hline "@n@" & 0 & false & false & false & false \\
\hline "@n@" & 2 & true & false & false & true \\
\hline "\%@@" & 1 & false & true & false & false \\
\hline "V@" & 1 & true & false & false & true \\
\hline
\end{tabular}

Table 2: An example state transformer and values of some expressions over the input states.

form of a state transformer. Informally, this state transformer is a function that, given an input state (i.e., valuation to variables) at the (potentially) faulty statement, produces an output state that would make every test in the test suite pass, including the failing ones. For passing tests, state transformers can be easily computed by simply observing input and output states during the execution of the tests. For failing tests, as we discuss in detail in Section 3.1. MintHint computes state transformers using dynamic symbolic execution and constraint solving [4]. Let us illustrate this step using the statement at line 70 . Because the statement is a conditional statement, MintHint uses a fresh boolean variable, say branch , $_{0}$, to represent the outcome of the branch predicate. That is, the statement is transformed into an assignment branch $_{0}=s\left[{ }^{*} i\right]==$ ENDSTR, in which the fault is on the RHS, and the branch predicate becomes the variable branch $_{0}$. Table 2 shows some entries in the state transformer of the statement: (1) the string array $\mathrm{S}$ and index expression $*_{i}$ in the input state and (2) the LHS variable branch in the output state. The values of all variables except branch $_{0}$ remain unchanged between a pair of input/output states, and hence are not shown again. The value of branch ${ }_{0}$ in the first row is obtained from a passing test by concrete execution and the remaining are obtained from failing tests by symbolic execution. These are the values that branch ${ }_{0}$ is expected to take in the repaired version, on the respective input states.

Step 3: Ranking of expressions. The goal of this step is to identify syntactic building blocks (i.e., expressions) for constructing an RHS expression compliant with the computed state transformer. To do so, MintHint searches the solution space, called the repair space, for expressions whose values over the input state of the state transformer are statistically correlated with the corresponding output values produced by the state transformer. More precisely, MintHint interprets correlation coefficients, which represent the numerical measure of the strength of a statistical correlation [8, 27], as the likelihood of the expression to occur in the repaired RHS. For our example, MintHint would compute correla-

\begin{tabular}{rlc}
\hline Rank & Expression & Likelihood \\
\hline 1 & $\mathrm{~s}\left[{ }^{*} \mathrm{i}+1\right]==$ ENDSTR & 0.62 \\
2 & $\mathrm{~s}\left[{ }^{*} \mathrm{i}+1\right]<=$ ENDSTR & 0.62 \\
& $\ldots$ \\
7 & $\mathrm{~s}\left[^{*} \mathrm{i}+1\right] !=$ ENDSTR & 0.62 \\
& $\ldots$ \\
23 & $\mathrm{~s}[1]==$ ENDSTR & 0.43 \\
& $\ldots$ \\
488 & $\mathrm{~s}\left[^{*} \mathrm{i}\right]==$ ENDSTR & 0 \\
& $\ldots$
\end{tabular}

Table 3: A partial list of expressions ranked by the likelihood of occurrence in the repaired RHS.

\begin{tabular}{|c|c|c|c|}
\hline Rank & Statement & Hint & Score \\
\hline 1 & 70 & $\begin{array}{l}\text { Replace } s\left[{ }^{*} i\right]==\text { ENDSTR } \\
\text { with } s\left[{ }^{*} i+1\right]==\text { ENDSTR }\end{array}$ & 1 \\
\hline 2 & 70 & $\begin{array}{l}\text { Replace } s\left[{ }^{*} i\right]==\text { ENDSTR } \\
\text { with } s[1]==\text { ENDSTR }\end{array}$ & 1 \\
\hline 3 & 70 & $\begin{array}{l}\text { Insert } s\left[{ }^{*} i+1\right]<=\text { ENDSTR } \\
\text { and Remove } s\left[{ }^{*} i\right]==\text { ENDSTR }\end{array}$ & 1 \\
\hline 4 & 77 & Retain the statement & 1 \\
\hline 5 & 80 & Retain the statement & 1 \\
\hline 6 & 502 & Retain the statement & 1 \\
\hline 7 & 70 & Insert $s\left[{ }^{*} i+1\right]$ != ENDSTR & 0.62 \\
\hline
\end{tabular}

Table 4: Repair hints synthesized by MintHint.

tions between the values of the candidate expressions and the expected value of branch $_{0}$ at line 70 . For some expressions from the repair space and the faulty RHS itself, Table 2 gives the values over the input states. ENDSTR is the null character indicating endof-string. As can be seen, the values of $s\left[{ }^{*} i+1\right]==$ ENDSTR are

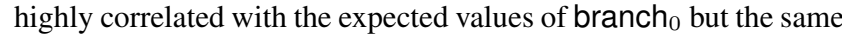
is not true for the other expressions. Table 3 shows some expressions in the repair space ranked by decreasing value of likelihood.

Step 4: Synthesis of repair hints. After producing a ranked list of expressions, MintHint analyzes this list to synthesize an $a c$ tionable list of repair hints. MintHint generates two types of hints: simple and compound. A simple hint is a single program transformation, whereas a compound hint is a set of program transformations. For our example, the hints synthesized by MintHint for some of the statements identified by the fault localization tool are given in Table 4 Note that MintHint may generate more than one hint for the same statement (e.g., statement 70 in Table 4). We now explain how MintHint generates such hints.

For simple hints, MintHint iteratively selects expressions from the repair space of each statement individually such that (1) their likelihood values are above a threshold and (2) the statistical correlation 
among themselves is low. That is, they form a set of expressions such that any one expression in the set is likely to appear in the repaired RHS. In our example, MintHint would select $S\left[{ }^{*} i+1\right] !=$ ENDSTR and $s[1]==$ ENDSTR. We discuss the algorithm for selection of expressions in detail in Section 3.2

After selecting these expressions, MintHint pattern matches [41] each of the selected expressions with the faulty RHS. Based on the edit distance between an expression and the subexpressions in the faulty RHS, MintHint determines the nature of the hint to be generated for the expression. If the edit distance is less than or equal to a threshold chosen heuristically (2 in our current formulation), MintHint suggests the replacement of the matching expression in the faulty RHS. Otherwise, if the edit distance is greater than the threshold, it suggests an insertion. Expression $s[1]==$ ENDSTR, for example, is at edit distance 2 from the faulty RHS's expression $s\left[{ }^{*} i\right]==$ ENDSTR, so MintHint synthesizes a replace hint for the RHS (position 2 in Table 4). The edit distance for $s\left[{ }^{*} i+1\right]$ != ENDSTR, conversely, is greater than 2, and MintHint synthesizes an insert hint for this expression (position 7 in Table 4 ).

If the edit distance of an expression selected through likelihood value is zero, that is, the expression already occurs as a subexpression in the faulty RHS, MintHint deems the RHS's subexpression as unlikely to be faulty and generates a retain hint for it. In our example, this happens for lines 80, 77, and 502 (positions 5, 4, and 6 , respectively). In all three cases, MintHint finds that the existing RHS is the most likely expression to appear in the repaired version and hence synthesizes the retain hints for the statements. In fact, there is no way to repair the fault through simple modifications of these statements. We can say that retain hints can help developers localize a fault by eliminating some spurious statements (i.e., false positives) returned by the fault localization tool.

MintHint ranks hints based on their score. Intuitively, the score indicates the confidence in the applicability of the hint and is derived from the likelihood values of the expressions involved. In the case of a replace hint, the score is the maximum of the likelihood of the expression being used for replacement and one minus the likelihood of the expression being replaced. For the replace hint at position 2 in Table 4 for instance, the score is $\max (0.43,1-0)$, where 0 is the likelihood value of the faulty RHS which is being replaced (see Table 3). For insert and retain hints, the score is the likelihood of the expression being inserted or retained (e.g., 0.62 for the insert hint at position 7 in Table 4). For a remove hint, it is one minus the likelihood of the expression being removed.

Whereas simple hints can address faults that can be repaired through a single syntactic transformation, compound hints can help repair more complex faults-faults that require more than one program transformation to be repaired or more refined pattern matching. Further, if the repair space contains only building blocks of the repaired RHS, but not the repaired RHS itself, then compound hints - obtained by algorithmically combining the building blocksbring the repair hints closer to the actual repair.

MintHint synthesizes compound hints by first computing sets of expressions such that (1) within each set, the likelihood values of the expressions are above a threshold and (2) all expressions in the repair space which are likely to appear in the repaired RHS together are included in the same set. This computation uses a variant of correlation coefficients, called partial correlation coefficients, and a more refined pattern matching. We differ the detailed discussion on this to Sections 3.1 and 3.2 In our example, MintHint would compute two such sets: $\left\{\mathrm{s}\left[{ }^{\star} \mathrm{i}+1\right]==\right.$ ENDSTR $\}$ and $\left\{\mathrm{s}\left[{ }^{*} \mathrm{i}+1\right]<=\right.$ ENDSTR $\}$. The selection criterion used here successfully identifies the required expression $s\left[{ }^{*} i+1\right]==$ ENDSTR. Each of these

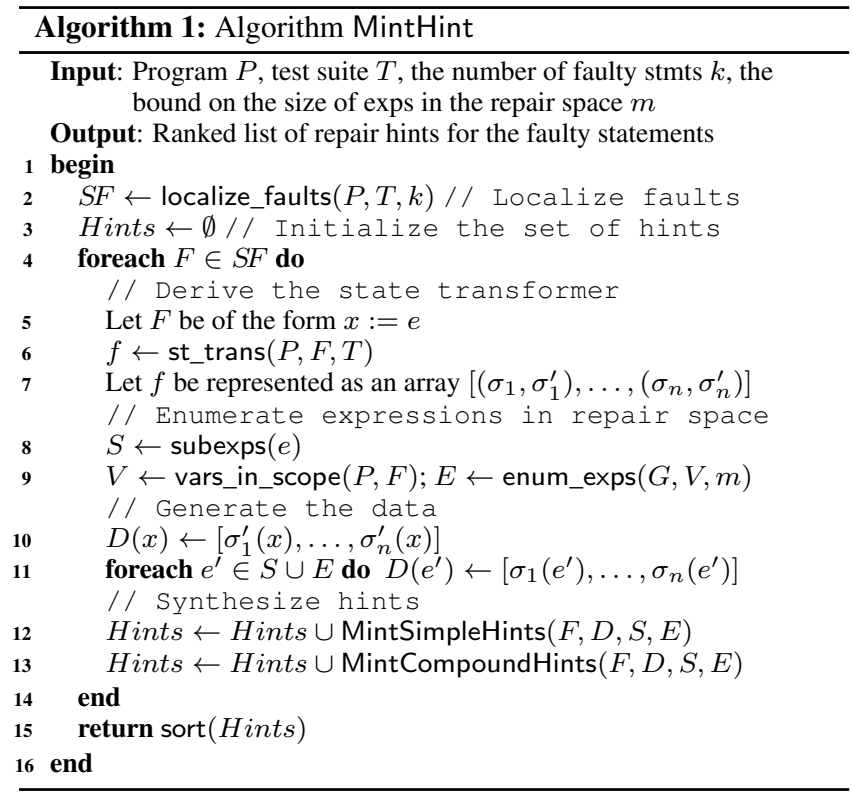

sets is singleton in this case. In general, however, the sets would contain more than one expression (e.g., see task 10 in Section 4.1).

After computing a set of expressions that may occur together in a repair, MintHint synthesizes actual compound hint using the edit distance of each expression in the set with the faulty RHS. For the first set in our example, $\left\{s\left[{ }^{*} i+1\right]==\right.$ ENDSTR $\}$, MintHint generates the replace hint at position 1 in Table 4 For the second set, $\left\{s\left[{ }^{*} i+1\right]<=\right.$ ENDSTR $\}$, it generates the hint at position 3 in Table 4 which has two constituent hints: an insert hint for the expression in the set together with a remove hint. MintHint adds the remove hint because, in this compound hint, there is no constituent hint which suggests retention or replacement of the faulty RHS.

To compute the score for a compound hint, MintHint computes the maximum of the scores of the constituent hints. After computing all hints and their scores, MintHint reports the hints to the developer, ordered by score (as in Table 4).

After getting the hints produced by MintHint, developers can manually apply the hints by modifying the potential faulty statement according to the hints. For example, in the case of a replace hint, developers will have to replace a subexpression with a suggested one. For a remove hint, developer will have to remove the subexpression in the hint. They will also have to suitably remove the operator(s) around the subexpression, or guess another expression to fill the hole, so as to obtain a well-formed resulting expression. Similarly, for an insert hint, developers will have to combine the subexpression in the hint with the existing expression and select an appropriate operator and place for insertion.

\section{Algorithm}

The MintHint algorithm is presented in Algorithm 1 As we discussed in the previous section, the input to the algorithm is a program $P$ and a test suite $T$ such that the program fails on at least one of the tests in $T$. MintHint also takes a threshold $k$ on the number of faulty statements to be considered for repair and a bound $m$ on the size of the expressions in the repair space.

The first step of MintHint (line 2 is to use the test suite to perform fault localization, as indicated by the function localize_faults. All statements in the fault localization list $S F$ are then processed in a loop (starting at line 4). Function st_trans at line 6 computes the state transformer for a faulty statement $F$. Sets $S$ and $E$, com- 
puted at lines 8 and 9 , contain the subexpressions of the faulty RHS and the extra expressions that should be considered when searching for repairs. The function $D$ maps expressions from the repair space and the LHS variable to their values according to the state transformer (similar to the column values in Table 2). Two separate algorithms, MintSimpleHints and MintCompoundHints are used for synthesizing simple and compound hints. Finally, the hints across all statements are sorted by their scores (line 15 .

In the following discussion, for simplicity, we consider statement $F$ to be of the form $x:=e$. A conditional statement can be rewritten in this form similar to the transformation of line 70 in Section 2 MintHint can handle cases in which the potentially faulty statement is a loop header of the form for(init, cond, upd), where init initializes the loop counter(s), cond is the loop termination condition, and upd is the update of the loop counter(s). In these cases, the fault could be in any of these three components, so MintHint spawns three different tasks-one each for init, cond, and upd. MintHint treats an assignment with a ternary RHS expression as a conditional statement in which the fault is in the branch predicate or in one of the assignments in the branches. MintHint can handle other constructs, such as switch or return, in analogous ways.

We now present the different steps of the algorithm (summarized above) in detail by discussing how MintHint goes (1) from the program's test suite to expressions that may help repair the fault and (2) from these expressions to actual repair hints.

\subsection{From Tests to Likelihood of Expressions}

This first part of the MintHint algorithm is based on symbolic and statistical analysis and is performed in several phases. We discuss each of these phases separately.

\subsubsection{Fault Localization}

Function localize_faults leverages an existing fault localization technique to compute a set $S F$ of potentially faulty statements. Any fault localization technique that requires only the program $P$ and a test suite $T$ can be used here (e.g., [1, 20, 25]). These techniques, called spectra-based techniques, gather runtime data on the execution of passing and failing tests and use data clustering techniques to rank statements by their likelihood of being faulty.

Given this ranked list, MintHint analyzes the top $k$ statements in the list, where $k$ is a threshold set by the user that can be increased progressively, if necessary. We recall that, analogously to other existing repair approaches (e.g., [29 31]), MintHint currently assumes that faults can be repaired by changing a single statement. Therefore, MintHint synthesizes repair hints for each statement $F$ in $S F$, the set of potentially faulty statements, independently.

\subsubsection{Derivation of State Transformers}

For each potentially faulty statement $F$, function st_trans derives a state transformer $f$ that, when substituted to $F$, makes the program produce the correct output for each test in $T . f$ is a function from program states to program states, where a program state $\sigma$ is a mapping from the variables in scope at the faulty statement to appropriately typed values. More formally, in Algorithm 1$] f$ is an array of pairs of input/output states: $f=\left[\left(\sigma_{1}, \sigma_{1}^{\prime}\right), \ldots,\left(\sigma_{n}, \sigma_{n}^{\prime}\right)\right]$ (line 7). Notationally, an unprimed state is an input state (at $F$ ), and a primed state is the corresponding output state.

Function $f$ is defined for states that can be witnessed at $F$, given the inputs in $T$. Tests in $T$ that do not execute $F$ are ignored when computing state transformers. For each passing test traversing $F$, MintHint runs the program and collects the input/output states at $F$. Conversely, for failing tests that traverse $F$, MintHint (1) makes the LHS variable $x$ symbolic, (2) uses a symbolic execution algorithm with constraint solving to obtain a value of $x$ that makes the program produce the correct output, and (3) reruns the program concretely using the so computed value for $x$ (instead of the original value of the RHS $e$ ). The input/output states at $F$ in this concrete execution give the mapping $f$ for the failing test. MintHint also handles the cases where the faulty statement is executed more than once in a passing or failing test.

Symbolic execution may fail to obtain a value for $x$ that results in the correct output. If all failing tests of $F$ still fail during symbolic execution (i.e., the program fails irrespective of $F$ ), it is possible that $F$ may not be the faulty statement. In such cases, MintHint generates a "retain the statement" hint. MintHint also sets timeout for symbolic execution and if the symbolic execution times out on every failing test within the timeout threshold then MintHint discards the statement and does not generate any hints.

In its subsequent phases, MintHint treats the computed state transformer $f$ for each potentially faulty statement $F$ as an operational specification for the repair, thus eliminating the need to have a formal specification.

\subsubsection{Ranking of Expressions}

Using the state transformer $f$ as a specification for statement $F$, MintHint ranks expressions in the repair space of $F$ according to their likelihood of occurring on the RHS of the repaired version of $F$. The repair space to be searched over can be obtained in several ways, such as by enumerating expressions over variables in scope or mining expressions that occur elsewhere in the program (similar to what is done in [29). Presently, MintHint uses the former approach. More precisely, function vars_in_scope, in Algorithm 1 computes the set $V$ of variables in scope at $F$. Then, function enum_exps enumerates the expressions of size up to $m$ (a userdefined threshold) over $V$ according to the grammar of expressions in the programming language in which $P$ is implemented. Let $E$ be the set of these expressions.

MintHint also includes in the repair space the set of subexpressions of $e$, the RHS expression, including $e$ itself. It does so because it evaluates whether they are likely to be faulty or not independent of the fault localization results. This set, which we call $S$, is computed by function subexps (line 8 . The repair space is thus defined as $E \cup S$.

We recall that a program state $\sigma$ is a mapping from variables to values. This mapping can be extended naturally to expressions. In particular, if $e \equiv e^{\prime}$ op $e^{\prime \prime}$ then $\sigma(e)=\llbracket o p \rrbracket\left(\sigma\left(e^{\prime}\right), \sigma\left(e^{\prime \prime}\right)\right)$. Let $D(x)=\left[\sigma_{1}^{\prime}(x), \ldots, \sigma_{n}^{\prime}(x)\right]$ be the values of the LHS variable $x$ over the output states defined by state transformer $f$ (line 10 . Similarly, for an expression $e^{\prime}$ in the repair space, let $D\left(e^{\prime}\right)=$ $\left[\sigma_{1}\left(e^{\prime}\right), \ldots, \sigma_{n}\left(e^{\prime}\right)\right]$ be the values of the expression $e^{\prime}$ over the input states defined by $f$ (line 11 .

Given the data $D(x)$ and $D\left(e^{\prime}\right)$, MintHint's goal is to find whether $x$ and $e^{\prime}$ are related with each other, that is, whether a change of value in one is accompanied by a change of value in the other. Because $D(x)$ contains the expected (correct), rather than current (erroneous), values of $x$ for the failing tests, the faulty RHS and its faulty subexpressions should not be highly related with $x$. Therefore, the expressions that are highly related with $x$ will be treated as building blocks for the repair. Of course, just because the fault localization tool marks a statement as potentially faulty, it does not mean that the statement is actually faulty. The statistical analysis performed by MintHint is discriminative enough so that, for a spuriously marked statement (i.e., a false positive), the existing RHS expression $e$ itself may appear as highly related to $x$.

Statistical Correlation. In statistics, the problem of finding whether two variables are related with each other is called statistical correlation. A correlation coefficient is a numerical measure of the strength of the correlation between two statistical vari- 
ables [8] 27. In our context, a statistical variable is either the LHS variable $x$ or an expression $e^{\prime}$. The (absolute) value of a correlation coefficient ranges over $[0,1]$. A correlation coefficient value close to 0 indicates that the variables are statistically uncorrelated, whereas values close to 1 indicate strong correlation.

There are a number of correlation coefficients that are used for identifying different types of correlations between variables (e.g., linear or monotonic correlations). In its current form, MintHint uses the Spearman coefficient [8]. This coefficient can be applied to any data domain for which there is a ranking function $r$ that can map the values in the data domain to a totally-ordered set. The coefficient is defined even if the data domains of the two variables are different. MintHint presently computes coefficients over three data domains: Booleans, integers, and ASCII encoding of characters. In general, there can be multiple variables in the system. We may want to compute the strength of the correlation between a pair of variables by eliminating the effect of a subset $\left\{c_{1}, \ldots, c_{m}\right\}$ of the other variables. This set is called a controlling set. The Spearman partial correlation coefficient is a variant of Spearman coefficient between a pair of variables with respect to a controlling set.

Example I. As an example of how correlation coefficients can be applied for reasoning about program constructs, consider a dataset generated by the assignment $y:=i+j * k-10$ over integer variables $i \in[1,2], j \in[1, \ldots, 5]$, and $k \in[1, \ldots, 25]$. The Spearman coefficient between the values of the (non-linear) subexpression $j * k$ and the values of the variable $y$ is 0.9997 . Clearly, the high coefficient value conforms to the fact that the term $j * k$ is, numerically, a dominant term on the RHS. Conversely, $i$ has a very small range and is not a dominant term on the RHS, so its coefficient is only 0.0230 . Nevertheless, $i$ is still a contributing term on the RHS. We can use partial correlation to assess the correlation between $y$ and $i$ by eliminating the effect of $j * k$. In this example, the value of the partial correlation between $y$ and $i$, with $j * k$ as the controlling set, is 0.9116 .

Complexity. The complexity of computing the Spearman coefficient between datasets of size $n$ is $O(n \log n)$. The complexity of computation of the Spearman partial coefficient is $O\left(n^{3}\right)$, for $n$ larger than $m$ where $m$ is the size of the controlling set.

Likelihood and Ranking of Expressions. The MintHint algorithm is based on the hypothesis that an expression $e^{\prime}$ is likely to occur on the RHS in the repaired version of $F$ iff it is highlycorrelated with $x$ on the dataset obtained from the state transformer which gives the expected, correct values of $x$ even for the failing tests. Based on this hypothesis, MintHint computes two measures for expressions: likelihood and partial likelihood.

The likelihood of an expression $e^{\prime}$ to occur in the repaired RHS, denoted by likelihood $\left(e^{\prime}\right)$, is the absolute value of the Spearman coefficient between $e^{\prime}$ and $x$ over the datasets $D\left(e^{\prime}\right)$ and $D(x)$ (obtained at lines 10 and 11. Given a set $L$ of expressions, the partial likelihood of $e^{\prime}$ to occur in the repaired RHS along with the expressions in $L$, denoted by $\mathrm{p}_{-}$likelihood $\left(e^{\prime}, L\right)$, is the absolute value of the Spearman partial correlation coefficient of $D\left(e^{\prime}\right)$ and $D(x)$ with $\left\{D\left(e^{\prime \prime}\right) \mid e^{\prime \prime} \in L\right\}$ as the data of the controlling set $L$.

Example II. Consider a faulty version $y:=i+j / k-10$ of the statement from Example I, where an incorrect operator / is used in place of $*$. Through symbolic execution, MintHint would compute the expected values of $y$ which would be close, if not identical, to the values that $y$ would take in the repaired program. With this data, the expression $j * k$ would get likelihood value of 0.9997 . On the other hand, the faulty subexpression $j / k$ would get a likelihood value of 0.2109 . For the sake of this example, these coefficients

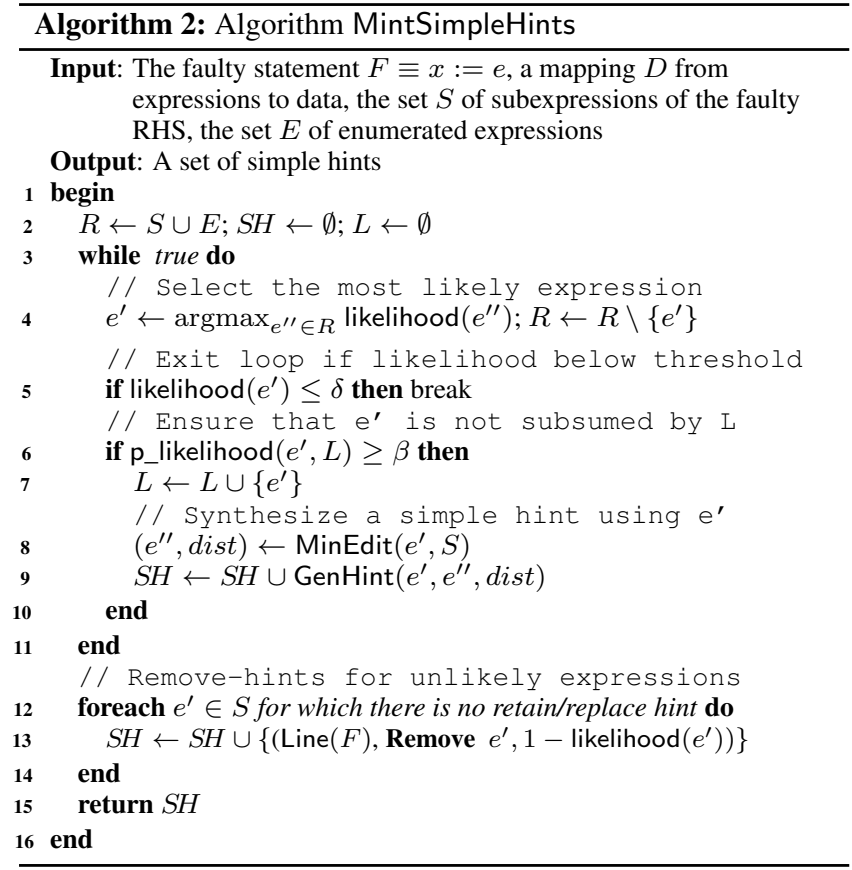

are computed wrt values of $y$ from Example I. MintHint would then use this information and apply pattern matching to generate a "Replace $j / k$ by $j * k$ " hint. This technique is discussed next.

\subsection{From Likelihood of Expressions to Hints}

In this second part, we discuss how MintHint utilizes the likelihood values of expressions computed in the first part of the algorithm to synthesize hints. Intuitively, MintHint synthesizes hints by performing syntactic pattern matching between the expressions that are likely to occur in the repaired RHS and the subexpressions of the faulty RHS, using different patterns to address different types of possible faults. Formally, a repair hint $h$ for a statement $F$ is a triple $(\ell, t, s)$ comprising the line number $\ell$ of statement $F$, a textual hint $t$, and the hint's score $s$. The keywords in the textual hint, shown in bold font, have their usual English meaning.

\subsubsection{Simple Hints}

MintSimpleHints (see Algorithm 2) synthesizes a set $\mathrm{SH}$ of simple hints given a faulty statement $F$, a dataset $D$ obtained from $F$ 's state transformer, subexpressions $S$ of the faulty RHS, and extra expressions $E$ over which to search. The algorithm also makes use of two thresholds $\delta$ and $\beta$, used respectively, for selecting expressions by likelihood and for checking partial likelihood of a candidate expression given the already selected expressions.

MintSimpleHints initializes the repair space $R$ to $S \cup E$, the set of simple hints $S H$ and the set of likely expression $L$ to the empty set (line 22. It then executes the loop starting at line 3 until the likelihood of expressions drops below the threshold $\delta$ (line 5). Within this loop, an expression $e^{\prime} \in R$ with the highest likelihood is selected and removed from $R$ (line 4 ). If the partial likelihood of $e^{\prime}$ with $L$ as the controlling set is above the threshold $\beta$ (line 6 ) then it is added to $L$. This check ensures that $e^{\prime}$ has sufficient statistical correlation with $x$ (the LHS) after taking out the effect of $L$, that is, $e^{\prime}$ is not subsumed by $L$. In the example in Section 2 once $s\left[{ }^{*} \mathrm{i}+1\right]$ != ENDSTR is added to $L$, all expressions until s[1] == ENDSTR (ranked 23) have partial correlation below the threshold $\beta=0.1$.

Now, MintSimpleHints generates a simple hint for $e^{\prime}$. To do so, it first invokes function MinEdit, which identifies the expression $e^{\prime \prime}$ from $S$ with minimal edit distance [41] from $e^{\prime}$ (line 8). Let dist be the minimal edit distance. Then, the algorithm invokes 
function GenHint, which synthesizes a simple hint based on the value of dist (line 9). If dist is below a given threshold, it generates hint "Replace $e^{\prime \prime}$ by $e^{\prime \prime}$. Conversely, if dist is above such threshold, it generates hint "Insert $e^{\prime \prime}$. For the former, the score of the hint is set to the maximum between the likelihood of $e^{\prime}$ and the unlikelihood of $e^{\prime \prime}$ (i.e., $1-\operatorname{likelihood}\left(e^{\prime \prime}\right)$ ). For the latter, the score is simply the likelihood of $e^{\prime}$. In the current formulation, MintHint uses threshold of 2 over the edit distance. This seems to capture errors resulting from incorrect operator, constant, variable, array index, and so on. Increasing the threshold may generate spurious replace hints and reducing it would produce two separate hints, respectively insert and remove, for $e^{\prime}$ and $e^{\prime \prime}$.

If dist $=0$ (i.e., $e^{\prime}$ already belongs to $S$ ), the algorithm generates hint "Retain $e^{\prime \prime}$ " and assigns to the hint a score equal to likelihood $\left(e^{\prime}\right)$. A special case of this is when $e^{\prime}$ is exactly the same as the RHS expression for which the algorithm generates a "retain the statement" hint. Finally, for the expressions that appear in the faulty RHS but not in set $L$, it generates remove hints in the loop at line 12 where function Line returns the line number of a statement. The score of a "Remove $e^{\prime \prime}$ ' hint is $1-$ likelihood $\left(e^{\prime}\right)$.

\subsubsection{Compound Hints}

Function MintCompoundHints, called in Algorithm 1 at line 13 . computes compound hints using the same inputs as MintSimpleHints. Due to space constraints, we do not typeset MintCompoundHints and simply explain the key similarities and differences between MintCompoundHints and Algorithm 2 Similar to Algorithm 2. it iteratively computes the set of likely expressions. However, it uses p_likelihood $\left(e^{\prime \prime}, L\right)$ in place of likelihood $\left(e^{\prime \prime}\right)$ at line 4 and the branch predicate at line 6 is replaced with true. The value of partial likelihood gives the measure of the likelihood of $e^{\prime \prime}$ to appear in the repaired RHS along with the expressions in $L$. As a consequence, the expressions that get added to the set $L$ are all those expressions which can occur together in the repaired RHS. In contrast, the expression selected at line 4 in Algorithm 2 is selected only based on its individual likelihood.

In general, MintCompoundHints can generate more than one set of likely expressions. More specifically, if multiple expressions have the highest partial correlation coefficient (at line 4 ) in the first iteration of the loop, then the algorithm would partition them into three sets based on their edit distance from the faulty RHS and its subexpressions: (1) equal to zero, (2) less than equal to 2 , and (3) more than 2. It initializes three sets of likely expressions by selecting one expression from each of the partitions above (if not empty). It then proceeds independently to select other expressions to add to each of them. (Conversely, when generating simple hints, for multiple expressions with the highest correlation coefficient, MintSimpleHints selects one expression at random without partitioning them by edit distance.) For example, in Section 2 the expression $s\left[{ }^{*} i+1\right]==$ ENDSTR is the only expression, among the seven expressions with the highest likelihood, which is at edit distance less than equal to 2 .

For each expression in the set $L$, MintCompoundHints generates a hint with the pattern matching logic used at line 9 in Algorithm 2 We call each of these hints, a constituent hint, of the compound hint. We say that a pair of constituent hints of a compound hint conflict if they refer to either the same or overlapping subexpressions of the faulty RHS. Two subexpressions of the faulty RHS overlap if their subtrees in the AST of the faulty RHS have some common node(s). Overlapping subexpressions can be identified by a simple walk over the AST. In order to ensure that no conflicting hints are generated, MintHint removes a subexpression and all expressions that overlap with it from the repair space as soon as the subexpression is added to set $L$. (Simple hints are independent of

\begin{tabular}{|l|c|c|}
\hline Program & LOC & \#Tasks \\
\hline print_tokens2 & 570 & 2 \\
\hline replace & 564 & 4 \\
\hline tcas & 173 & 4 \\
\hline
\end{tabular}

Table 5: Description of tasks.

each other and hence are permitted to conflict.) Finally, analogous to the loop at line 12 in Algorithm 2 MintCompoundHints generates remove hints for the subexpressions of the faulty RHS for whom there is no retain or replace hint synthesized earlier.

There could be multiple simple hints that suggest retention of different subexpressions of the RHS. In these cases, these hints are clustered into one single compound hint. The score of a compound hint is the maximum of the scores of the constituent hints.

\section{Evaluation}

To assess the effectiveness of our approach, we implemented MintHint to synthesize repair hints for $\mathrm{C}$ programs and performed an empirical evaluation. Our implementation uses Zoltar [20] for fault localization, KLEE [4] for dynamic symbolic execution with constraint solving, and Matlah ${ }^{1}$ for statistical analysis.

Our evaluation consists of two parts. We performed a user study to evaluate whether MintHint improves developers' productivity. The user study is augmented with the application of hints on a case study. We investigate the following research questions:

RQ1: Usefulness of hints - Can MintHint produce useful hints, enabling developers to repair programs more effectively?

RQ2: Robustness - How does MintHint perform when the repair space is incomplete (i.e., does not contain the repaired version of the faulty expression) and is supplied imprecise data?

RQ3: Performance and scalability - How does MintHint scale to large programs, state transformers, and repair spaces?

\subsection{User Study}

Experimental Setup. We performed fault localization on the programs from the Siemens suite [19]. The Siemens suite consists of a few programs with multiple faulty versions for each of them. Table 5 lists the programs and the number of faulty versions that were selected as tasks. In all, 10 tasks were selected. In the user study, each user was required to work on two independent tasks. To keep each task manageable within $2 \mathrm{~h}$, for each task, only the top 5 statements identified by Zoltar as potentially faulty were presented to the users. For each of the chosen tasks, the actual faulty statement belonged to this list. The tasks represent a diverse collection of faults (see Table 6). For each program and candidate faulty statement, the state transformers were obtained for failing tests through symbolic execution with the timeout of $5 \mathrm{~m}$ per test. In one of the tasks, symbolic execution of many failing tests timed out. Consequently, the data from the passing tests was seen to bias the results. Therefore, for that task, only half of the passing tests were used.

Of the 10 users who participated in our study, 8 were working professionals and 2 were graduate students (with prior industry experience) - none affiliated with our research group. Of these, 8 stated that they had moderate to high expertise with $\mathrm{C}$ programming/debugging with everyone having at least 1 year of experience in $\mathrm{C}$ programming. For each task, the input/output specifications of methods, and meaning of variables and named constants were presented as comments in the source code. In addition, each user was provided a test suite of 10 passing tests and was given $15 \mathrm{~m}$ to study the program before starting with program repair.

There were two phases of the user study. In the control phase, the users were given the fault localization information and test suite. In the experimental phase, in addition, they were given the repair

\footnotetext{
http://www.mathworks.com/products/matlab/
} 


\begin{tabular}{|c|c|c|c|c|c|c|}
\hline Task & Program-Version & Nature of fault & Type of the most useful hint & $\begin{array}{c}\text { Rank of the most } \\
\text { useful hint }\end{array}$ & \#Total hints & $\begin{array}{l}\text { \#Stmts with only } \\
\text { "Retain the stmt" hints }\end{array}$ \\
\hline 1 & print tokens2-v6 & Incorrect array index & Remove & 10 & 31 & - \\
\hline 2 & replace-v7 & Superfluous expression & Compound (Remove + others) & 4 & 13 & 1 \\
\hline 3 & print tokens2-v7 & Superfluous expression & Compound (Remove + others) & 4 & 8 & - \\
\hline 4 & replace-v18 & Missing expression & Compound (Insert + others) & 1 & 3 & 1 \\
\hline 5 & replace-v23 & Incorrect array index & Replace & 1 & 6 & 3 \\
\hline 6 & $\overline{\text { tcas-v28 }}$ & Incorrect operator & Replace & 6 & 26 & - \\
\hline 7 & replace-v8 & Missing expression & Compound (Retain + others) & 1 & 6 & 2 \\
\hline 8 & tcas-v2 & Incorrect constant & Replace & 7 & 13 & 1 \\
\hline 9 & $\overline{\text { tcas-v1 }}$ & Incorrect operator & Replace & 2 & 11 & 3 \\
\hline 10 & $\overline{\text { tcas-v12 }}$ & Incorrect operator & Compound (Retain + others) & 11 & 14 & - \\
\hline
\end{tabular}

Table 6: Description of faults in the user study and of useful hints identified by the users: The tasks that could not be finished in the control phase (without hints) are underlined. All the tasks were completed in the experimental phase (with hints).

\begin{tabular}{|c|c|c|}
\hline & $\begin{array}{l}\text { Control phase } \\
\text { (without hints) }\end{array}$ & $\begin{array}{l}\text { Experimental phase } \\
\text { (with hints) }\end{array}$ \\
\hline \multicolumn{3}{|c|}{ Quantitative Analysis } \\
\hline Successful localization & $8 / 10$ & $10 / 10$ \\
\hline Successful repair & $6 / 10$ & $10 / 10$ \\
\hline Avg. time to repair & $91 \mathrm{~m}+4$ timeouts & $29 \mathrm{~m}$ (no timeouts) \\
\hline Avg. speedup (excl. timeouts) & NA & $5.8 \mathrm{x}$ \\
\hline \multicolumn{3}{|c|}{ Qualitative Analysis (Ratings given by the users) } \\
\hline \multirow{3}{*}{ Difficulty of localization } & Easy: 2 & Easy: 6 \\
\hline & Moderate: 6 & Moderate: 3 \\
\hline & Difficult: 2 & Difficult: 1 \\
\hline \multirow{3}{*}{ Difficulty of repair } & Easy: 3 & Easy: $7 \quad(+4)$ \\
\hline & Moderate: 5 & Moderate: 1 \\
\hline & Difficult: 2 & Difficult: 2 \\
\hline
\end{tabular}

Table 7: Results of the user study.

\begin{tabular}{|l|l|c|}
\hline & Yes & No \\
\hline Incompleteness of repair space & 5 & 5 \\
\hline Noise in state transformers obtained from tests & 7 (max. 27\%) & 3 \\
\hline
\end{tabular}

Table 8: Count of tasks in the user study wrt incompleteness of the repair space and noise in state transformers.

hints. Each user worked on a single task in a phase and was given $2 \mathrm{~h}$ to complete the task. A task was considered to be complete if the repaired program passed all the tests. The users chose the programs for the control phase by drawing lots. We had mapped each task in the control phase to a task in the experimental phase with the objective of avoiding a user working on the same task or another version of the same program in both phases. A presentation was made to them to explain the meaning of different types of repair hints. The users were provided Linux based machines with a debugger, text editors and IDEs, and the standard command line utilities.

(RQ1) Usefulness of hints. Table 7 summarizes the results of the user study. In the control phase (without hints), out of 10 tasks, the users could localize the faults to the actual faulty statement in 8 cases but only managed to repair the programs in 6 cases. On the contrary, in the experimental phase (with hints), the users could perform localization and repair in all the 10 cases. Further, the average time taken to repair a fault was $91 \mathrm{~m}$ in the control phase (excl. the 4 timeouts), whereas it was $29 \mathrm{~m}$ in the experimental phase. The average speedup obtained with the use of hints, for the 6 tasks that were completed in both the phases, was equal to $5.8 \mathrm{x}$. The users were asked to rate the difficulty level of fault localization and repair for their tasks as easy, moderate, or difficult. The ratings, for the same set of tasks, differ across the two phases. Notably, with the hints, 4 more tasks were rated by the users as easy. These qualitative ratings corroborate the quantitative results presented above.

The users uniformly mentioned that the hints were useful and were asked to indicate the hint that was most useful to them. The ranks of the most useful hints in Table 6 are over the entire list of hints for all statements presented to the user for that task. Note that MintHint may produce multiple hints for a faulty statement. The most useful hints were all in top 10 except for one case. The last column in Table 6 gives the number of statements for which only a "retain the statement" hint was generated. These are the statements that are classified by MintHint as unlikely to be faulty. Across the 10 tasks and 50 statements in the fault localization lists, 40 statements are likely to be false positives ( 10 are true positive, i.e., definitely faulty). Out of these, 11 statements (more than $25 \%$ ) are eliminated by MintHint. This contributes greatly to ease of localization. We believe that there is no obvious way to rectify these statements. In fact, no user in the study (even in the control phase) came up with a repair for any of these statements.

For 6 tasks (tasks 2-6 and 9), the most useful hint had the precise information required to repair the fault. For task 10, the faulty RHS was of the form exp1 || exp2 and the compound hint suggested that both exp1 and exp2 be retained but did not say the same for the entire RHS. The user therefore suspected that the operator was incorrect and correctly replaced it with \&\&. For task 1 , the hint suggested only removal of the incorrect expression. The user had to come up with the substitute expression. In task 7, the hint did not suggest the expression to be inserted. The user mentioned that the hints helped mainly in localizing the fault. This is possible because MintHint eliminates 2 other statements in this case (see the last column for task 7 in Table 6. In the case of task 8, the replace hint did not suggest the required (named) constant to be used. Nevertheless, the user observed that after substituting the new expression suggested in the hint, many failing tests started passing and subsequently inferred the right constant manually. Of these, tasks 5, 8, 9, and 10 were not completed in the control phase.

The number of total hints per task given in Table 6 depends on the following factors: (1) The thresholds on correlation coefficients which determine how many expressions end up in the set of likely expressions, thereby, also affect the number of hints. Across all tasks, the thresholds on correlation coefficient and partial correlation coefficient for generation of simple hints were 0.4 and 0.1 respectively. For compound hints, it was 0.6. (2) If the symbolic execution times out on all failing tests for a statement then the hint generation algorithm is not run for that statement (see Section 3.1.2.

Though the study is not large enough to measure the difference with statistical significance, the results suggest that repair hints can contribute significantly in improving developers' productivity and even the feasibility of repair (within limited time).

(RQ2) Robustness. In practice, it is difficult to estimate the syntactic space to search for a complete repair. In our experiments, for each statement, apart from the subexpressions of the potentially faulty expression, expressions of size up to 4 (over the variables in 
\#Exprs in repair space

(\#Columns of the data matrix)

\begin{tabular}{|c|c|c|c|c|}
\hline & & & & \\
\hline Size of state & & Upto $5 \mathrm{k}$ & Upto $10 \mathrm{k}$ & $>10 \mathrm{k}$ \\
\hline former & Upto $1 \mathrm{k}$ & $<1 \mathrm{~m}$ & $<1 \mathrm{~m}$ & $<1 \mathrm{~m}$ \\
\hline & Upto $10 \mathrm{k}$ & $<1 \mathrm{~m}$ & $<5 \mathrm{~m}$ & $<5 \mathrm{~m}$ \\
\hline & $>10 \mathrm{k}$ & $<1 \mathrm{~m}$ & $<5 \mathrm{~m}$ & $\approx 1 \mathrm{~h}$ \\
\hline
\end{tabular}

Table 9: Performance and scalability chart: Tasks from the user study belong to all cells except cell at position $(3,2)$. Tasks from the case study (Section 4.2) belong to the shaded cells.

scope at the statement) were added to the repair space. The size of an expression is the number of nodes in its abstract syntax tree (AST). For expressions involving arrays, an occurrence of an array with the index expression is counted as size 1 and the index expressions themselves can go up to size 4 . We call a repair space complete only if the repaired version of the expression belongs to it. For example, in task 10, the repaired expression exp1 \&\& exp2 was not in the repair space.

MintHint derives state transformers for failing tests by symbolic execution. In some cases, the derived constraints may have multiple satisfying assignments but not all of them can be observed in the execution of the repaired version of the program. The constraint solver may pick any one of them. For passing tests, the state transformer is obtained by concrete execution. Even though the test passes, the value generated by the faulty expression may not be observed in the repaired version. These situations make the resulting data, which is used as a specification, imperfect (noisy) and may in general invalidate the applicability of a repair.

Table 8 gives the count of tasks which had noise in the state transformers and where the repair space was incomplete. This information is provided only for the actual faulty statement. To estimate the amount of noise in the data, we first obtain the noise-free state transformer of the (known) repaired version independently by concrete execution over the test suite. An entry in the state transformer obtained over the faulty version is classified as noisy if it does not belong to the noise-free state transformer. There were 7 tasks with noisy data with the maximum of $27 \%$ noise in one of them and there were 5 tasks where the actual repaired expression did not belong to the repair space. Nevertheless, the successful completion of the tasks in the user study indicates that useful hints could be synthesized even in these challenging cases. The key reasons for this are (1) the use of statistical correlation which is robust in presence of noise and (2) the ability of MintHint to synthesize compound hints from building blocks. In particular, the repair spaces were incomplete for tasks $2-4,7$, and 10 , and as Table 6 shows, in each of these cases, the most useful hint was a compound hint.

(RQ3) Performance and scalability. The complexity of statistical correlation computation dominates the cost of hint generation (see Section 3.1.3 for discussion on its complexity). It works on a two-dimentional matrix where the number of rows is equal to the size of the state transformer (the number of input/output pairs) and the number of columns is equal to the number of expressions in the repair space. Table 9 gives the performance and scalability chart summarizing all runs of the hint generation algorithm for all tasks and faulty statements in the user study. Despite the large datasets, the hint generation algorithm scales well. Zoltar took slightly over $2 \mathrm{~m}$ on an average for fault localization. Except for a few statements, KLEE ran to completion on all the failing tests within $5 \mathrm{~m}$ (the timeout set by us). The timings are measured on a desktop with Intel i5 CPU@3.20 GHz and 4GB RAM.

\subsection{A Case Study}

Experimental Setup. We applied MintHint on a commonly used Unix utility program, the stream editor sed obtained from

\begin{tabular}{ccclccc}
\hline $\begin{array}{c}\text { Task Version- } \\
\text { Fault }\end{array}$ & Nature of fault & $\begin{array}{l}\text { Type of } \\
\text { useful } \\
\text { hint }\end{array}$ & $\begin{array}{c}\text { Rank of \#Total } \\
\text { useful } \\
\text { hint }\end{array}$ & $\begin{array}{c}\text { \#Stmts with } \\
\text { "Retain stmt" } \\
\text { hint only }\end{array}$ \\
\hline 1 & v2-f1 & Incorrect operator & Replace & 1 & 11 & 10 \\
2 & v3-f4 & Incorrect constant & - & - & 11 & 3 \\
3 & v3-f6 & Incorrect constant & Replace & 1 & 10 & 9 \\
4 & v5-f1 & Incorrect operator & Replace & 13 & 13 & 12 \\
5 & v6-f1 & Incorrect operators & Compound & 2 & 4 & - \\
6 & v6-f2 & \multicolumn{3}{r}{ Symbolic execution times out with $15 \mathrm{~m}$ threshold } \\
\hline
\end{tabular}

Table 10: Description of faults and hints in the case study.

\begin{tabular}{|l|l|c|}
\hline & Yes & No \\
\hline Incompleteness of repair space & 1 & 4 \\
\hline Noise in state transformers obtained from tests & 4 (max. 97\%) & 1 \\
\hline
\end{tabular}

Table 11: Count of tasks in the case study wrt incompleteness of the repair space and noise in state transformers.

the SIR repository [12]. sed is a reasonably large program with over $14 \mathrm{~K}$ LOC and 250 functions. The SIR repository consists of several versions of sed seeded with different faults. We performed fault localization on them using Zoltar. Table 10 lists the versions and fault-IDs of the programs that were selected as tasks. In all, 6 tasks were selected. The top 15 statements identified by Zoltar as potentially faulty were considered for repair. For each of the chosen tasks, the actual faulty statement belonged to this list. For each version and candidate faulty statement, the state transformers were obtained for failing tests through symbolic execution with the timeout of $5 \mathrm{~m}$ per test for the first three tasks and $15 \mathrm{~m}$ for the remaining. In the sixth task, symbolic execution timed out for all failing tests and hence it was not subjected to further analysis.

(RQ1) Usefulness of hints. For each of the tasks, Table 10 shows the nature of the fault and the type of the most useful hint. For tasks 1, 3, and 4, a replace hint synthesized by MintHint when applied, immediately lead to success on both passing and failing tests. Task 5 consists of two faults in the same statement. A compound hint suggested removal of two subexpressions and retention of the non-faulty subexpression. We removed the subexpressions identified by MintHint for removal and obtained well-formed expressions by removing operators around them. This change however lead to only a partial repair since MintHint did not generate the expressions that should be used for replacing the faulty ones. With this change, all the previously passing tests continued to pass and several previously failing tests too started to pass. In task 2, MintHint did not produce any useful hint for the faulty statement (due to excessive noise, as discussed below).

Interestingly, MintHint generated only "retain the statement" hints for many statements identified by Zoltar. Across the 5 tasks and 75 statements in the fault localization lists, 70 statements are likely to be false positives ( 5 are true positive, i.e., definitely faulty). Out of these, 34 statements (more than 45\%) are eliminated by MintHint. In fact, in tasks 1,3 , and 4 , retain were the only other type of hints apart from the replace hint required for program repair. We studied the faulty statements which had only the retain hints but could not identify a way to change them to repair the faults.

This study gives preliminary evidence that MintHint can be applied to large programs with not so accurate fault localization lists.

(RQ2) Robustness. Repair spaces were constructed in a manner similar to the user study and with the same bound on expression sizes. As shown in Table 11, the repair space searched by MintHint contained the repaired version of the faulty RHS in all but one case. The data obtained for the failing tests however contained noise in all cases except one. Our approach for estimating the amount of 
noise is explained in Section 4.1 In one case (task 2), the noise was $97 \%$ and MintHint could not produce any useful hint.

(RQ3) Performance and scalability. In Table 9 the shaded cells denote the time taken by MintHint for statistical correlation analysis and hint generation for the tasks in the case study. No task took more than $5 \mathrm{~m}$ for these steps. Fault localization finished within $1 \mathrm{~m}$ on an average for these tasks. While symbolic execution completed within $5 \mathrm{~m}$ on most failing tests for the first three tasks it required $15 \mathrm{~m}$ for most cases for tasks 4 and 5. However, even with $15 \mathrm{~m}$ threshold, it timed out for all failing tests and all potentially faulty statements for the sixth task.

\subsection{Limitations and Threats to Validity}

One of the main limitations of our approach is its reliance on symbolic execution for deriving state transformers, which is a complex and expensive technique. However, these techniques are becoming increasingly efficient, and many of their practical limitations are being addressed (e.g., [13 36]). Moreover, as we discuss in Section 6, we plan to investigate alternative, less expensive ways to build state transformers. Further, it is technically difficult to obtain only those values which can be observed in the repaired program through symbolic execution. This makes the state transformers noisy. Due to the statistical reasoning applied in MintHint, it produced useful hints even in presence of noise in many cases. The so-called measurement errors leading to noisy data are common in other application domains as well and a large body of work, commonly called outlier detection, exists to deal with them (see [5] for a survey). We plan to investigate applications of these techniques to further improve tolerance of MintHint to noise.

Like every empirical evaluation, ours too has potential threats to validity. Threats to internal validity for the user study include selection bias where the users working on the same task in control and experimental phases may have different expertise and testing bias where activities before the study may affect the outcome. Only two users had indicated low expertise with $\mathrm{C}$ programming, to prevent selection bias, we paired them in such a way that their tasks were interchanged in the two phases. Other users picked their tasks randomly. To mitigate testing bias, we ensured that no user worked on the same task or two faults of the same program, in the two phases of the user study. Further, we only made a presentation about the meaning of repair hints to them and did not provide any hands-on tutorial. There may be faults in our implementation that might have affected our results. To address this threat, we manually checked many of our results and did not encounter any error.

Threats to external validity arise because our results may not generalize to other group of developers (in the case of user study) and program repair tasks. In the user study, we ensured that the users did not have any prior experience with the programs used as repair tasks. While this ensures a level playing field, it leaves out users who might have better familiarity with the programs. The tasks in the user study were not hand-picked. We applied a welldefined criterion for their selection. The programs in Siemens suite were sorted by the rank of the actual faulty statement in the respective localization lists. Within each rank, the program-fault names were then sorted in the lexicographic order and finally, the first two tasks at each fault localization rank were selected. Similarly, the tasks in the case study, involving sed, consist of all tasks from the SIR repository [12] with only a single faulty statement such that the statement occurs within top 15 statements returned by a third-party fault localization tool, Zoltar. A few tasks could not be included because of limitations of the symbolic execution tool. The performance of MintHint is a function of the test suite also. It will take a much larger evaluation to ascertain how the quality of tests affects the quality of repair hints. It is however important to note that the tasks and test suites we used were also used in numerous previous papers in the area (e.g., 26, 31, 43]). Nevertheless, the number of tasks considered is small, so our findings may not generalize to other programs or faults. We plan to perform an additional extensive empirical evaluation to confirm our current results.

\section{Related work}

Early work by Arcuri [2], later extended by Le Goues and colleagues [29], proposes the use of genetic programming to automatically generate repairs that make failing test cases pass and do not break any passing test case. Debroy and Wong propose a similar approach, but based on the use of mutation [10].

Other approaches rely on the use of program specifications to repair the code. Pei and colleagues propose an approach for finding program repairs given program contracts [32 40]. Jobstmann, Griesmayer, and Bloem use a game theoretical approach for identifying repairs that satisfy a linear-temporal-logic specification of the program to be repaired [24]. Gopinath, Malik, and Khurshid's approach builds a SAT formula that encodes the constraints imposed by the specification on the program behavior and, if the formula is satisfiable, derives a repair from the SAT solution [14]. Konighofer and Bloem present a template-based approach that, given a faulty program and a specification, performs a symbolic analysis of program inputs and template parameters to generate a repair [28]. He and Gupta propose a technique that computes the weakest preconditions along a failing trace and compares the computed conditions with functions' pre- and post-conditions to find and correct faults [18]. Logozzo and Ball's approach generates verified program repairs from failed verification checks of programs that have developer-supplied modular specifications [30].

Yet other approaches leverage the existing test suite to infer specifications and generate repairs accordingly. PACHIKA [9] models a program's behavior for passing and failing test cases and generates a repair based on the differences between these models of correct and incorrect behavior. In a recent paper, Nguyen and colleagues propose the SemFix approach that combines angelic debugging [6] and program synthesis [22] to automatically identify program repairs [31]. BugFix [21] shares with our approach the idea of providing developers with "bug-fix suggestions", rather than actual repairs. Unlike our approach, however, BugFix generates suggestions using a machine-learning approach based on knowledge acquired from previous bug repairs.

Finally, some approaches perform program repair in specific domains, such as repairs for data structures [11], web application repairs [35], and repairs targeted at security vulnerabilities [33] or concurrency faults [23].

\section{Conclusions and Future Work}

We presented MintHint, a novel technique for semi-automated program repair. The key novelty of our approach is that it combines symbolic, statistical, and syntactic reasoning to synthesize repair hints, given only the faulty program and a test suite. Our evaluation of MintHint provides initial but strong evidence that our approach is effective. An interesting direction for future work is the investigation of how repair hints could be used to further automate the program-repair process. For example, we envision that hints could be used to inform program synthesis (e.g., [16, 17, 22]) or sketching (e.g., [37, 38]). In the future, we will extend our technique so that it can handle the more challenging case of faults involving multiple statements. Finally, in order to improve performance of MintHint, we plan to investigate (1) alternative, more efficient techniques for 
building operational specifications and (2) outlier detection mechanisms to further improve tolerance of MintHint to noise.

\section{References}

[1] R. Abreu, P. Zoeteweij, and A. J. C. v. Gemund. An Evaluation of Similarity Coefficients for Software Fault Localization. In PRDC, pages 39-46, 2006.

[2] A. Arcuri. On the Automation of Fixing Software Bugs. In ICSE Companion, pages 1003-1006, 2008.

[3] T. Ball, M. Naik, and S. K. Rajamani. From Symptom to Cause: Localizing Errors in Counterexample Traces. In POPL, pages 97-105, 2003.

[4] C. Cadar, D. Dunbar, and D. R. Engler. KLEE: Unassisted and Automatic Generation of High-Coverage Tests for Complex Systems Programs. In OSDI, pages 209-224, 2008.

[5] V. Chandola, A. Banerjee, and V. Kumar. Outlier detection: A survey. ACM Computing Surveys, 2007.

[6] S. Chandra, E. Torlak, S. Barman, and R. Bodik. Angelic debugging. In ICSE, pages 121-130, 2011.

[7] H. Cleve and A. Zeller. Locating causes of program failures. In ICSE, pages 342-351, 2005.

[8] H. Cramér. Mathematical Methods of Statistics. Princeton Landmarks in Mathematics and Physics. Princeton University Press, 1945.

[9] V. Dallmeier, A. Zeller, and B. Meyer. Generating fixes from object behavior anomalies. In ASE, pages 550-554, 2009.

[10] V. Debroy and W. E. Wong. Using Mutation to Automatically Suggest Fixes for Faulty Programs. In ICST, pages 65-74, 2010.

[11] B. Demsky, M. D. Ernst, P. J. Guo, S. McCamant, J. H. Perkins, and M. Rinard. Inference and Enforcement of Dta Structure Consistency Specifications. In ISSTA, pages 233244, 2006.

[12] H. Do, S. G. Elbaum, and G. Rothermel. Supporting controlled experimentation with testing techniques: An infrastructure and its potential impact. Empirical Software Engineering: An International Journal, 10(4):405-435, 2005.

[13] P. Godefroid, A. V. Nori, S. K. Rajamani, and S. D. Tetali. Compositional may-must program analysis: Unleashing the power of alternation. In ACM Sigplan Notices, volume 45, pages 43-56, 2010.

[14] D. Gopinath, M. Z. Malik, and S. Khurshid. Specificationbased program repair using sat. In TACAS, pages 173-188, 2011.

[15] A. Groce, D. Kroening, and F. Lerda. Understanding Counterexamples with Explain. In $C A V$, pages 453-456, 2004.

[16] S. Gulwani. Automating String Processing in Spreadsheets using Input-Output Examples. In PLDI, pages 317-330, 2011.

[17] S. Gulwani, V. A. Korthikanti, and A. Tiwari. Synthesizing Geometry Constructions. In PLDI, pages 50-61, 2011.

[18] H. He and N. Gupta. Automated debugging using path-based weakest preconditions. In FASE, pages 267-280, 2004.

[19] M. Hutchins, H. Foster, T. Goradia, and T. Ostrand. Experiments of the effectiveness of dataflow- and control flow-based test adequacy criteria. In ICSE, pages 191-200, 1994.

[20] T. Janssen, R. Abreu, and A. J. C. van Gemund. Zoltar: A toolset for automatic fault localization. In ASE, pages 662664. IEEE Computer Society, 2009.

[21] D. Jeffrey, M. Feng, N. Gupta, and R. Gupta. Bugfix: A Learning-based Tool to assist Developers in Fixing Bugs. In ICPC, pages 70-79, 2009.
[22] S. Jha, S. Gulwani, S. A. Seshia, and A. Tiwari. Oracle-guided Component-based Program Synthesis. In ICSE, pages 215224, 2010.

[23] G. Jin, W. Zhang, D. Deng, B. Liblit, and S. Lu. Automated Concurrency-bug Fixing. In OSDI, pages 221-236, 2012.

[24] B. Jobstmann, A. Griesmayer, and R. Bloem. Program Repair as a Game. In $C A V$, pages 287-294, 2005.

[25] J. A. Jones, M. J. Harrold, and J. Stasko. Visualization of test information to assist fault localization. In ICSE, pages 467-477, 2002.

[26] M. Jose and R. Majumdar. Cause clue clauses: error localization using maximum satisfiability. In $P L D I$, pages 437-446, 2011.

[27] M. Kendall and J. Gibbons. Rank correlation methods. A Charles Griffin Book. E. Arnold, 1990.

[28] R. Konighofer and R. Bloem. Automated Error Localization and Correction for Imperative Programs. In FMCAD, pages 91-100, 2011.

[29] C. Le Goues, T. Nguyen, S. Forrest, and W. Weimer. Genprog: A Generic Method for Automatic Software Repair. IEEE Trans. on Software Engineering, pages 54 -72, 2012.

[30] F. Logozzo and T. Ball. Modular and Verified Automatic Program Repair. In OOPSLA, pages 133-146, 2012.

[31] H. D. Nguyen, D. Qi, A. Roychoudhury, and S. Chandra. SemFix: Program Repair via Semantic Analysis. In ICSE, 2013.

[32] Y. Pei, Y. Wei, C. A. Furia, M. Nordio, and B. Meyer. Codebased Automated Program Fixing. In ASE, pages 392-395, 2011.

[33] J. H. Perkins, S. Kim, S. Larsen, S. Amarasinghe, J. Bachrach, M. Carbin, C. Pacheco, F. Sherwood, S. Sidiroglou, G. Sullivan, W.-F. Wong, Y. Zibin, M. D. Ernst, and M. Rinard. Automatically Patching Errors in Deployed Software. In SOSP, pages 87-102, 2009.

[34] M. Renieris and S. P. Reiss. Fault localization with nearest neighbor queries. In $A S E$, pages 30-39, 2003.

[35] H. Samimi, M. Schäfer, S. Artzi, T. Millstein, F. Tip, and L. Hendren. Automated Repair of HTML Generation Errors in PHP Applications using String Constraint Solving. In ICSE, pages 277-287, 2012.

[36] N. Sinha, N. Singhania, S. Chandra, and M. Sridharan. Alternate and learn: Finding witnesses without looking all over. In $C A V$, pages 599-615, 2012.

[37] A. Solar-Lezama, R. Rabbah, R. Bodík, and K. Ebcioğlu. Programming by Sketching for Bit-streaming Programs. In PLDI, pages 281-294, 2005.

[38] A. Solar-Lezama, L. Tancau, R. Bodík, S. A. Seshia, and V. A. Saraswat. Combinatorial sketching for finite programs. In ASPLOS, pages 404-415, 2006.

[39] I. Vessey. Expertise in Debugging Computer Programs. International Journal of Man-Machine Studies: A process analysis, pages 459-494, 1985.

[40] Y. Wei, Y. Pei, C. A. Furia, L. S. Silva, S. Buchholz, B. Meyer, and A. Zeller. Automated Fixing of Programs with Contracts. In ISSTA, pages 61-72, 2010.

[41] K. Zhang, R. Statman, and D. Shasha. On the editing distance between unordered labeled trees. Inf. Process. Lett., 42(3):133-139, 1992.

[42] X. Zhang, N. Gupta, and R. Gupta. Locating Faults through Automated Predicate Switching. In ICSE, pages 272-281, 2006.

[43] X. Zhang, N. Gupta, and R. Gupta. Locating faults through automated predicate switching. In ICSE 2006, pages 272- 
281, 2006. 